\title{
Effect of Aqua Therapy Exercises on Postmastectomy Lymphedema: A Prospective Randomized Controlled Trial
}

\author{
Khadra Mohamed Ali, $\mathrm{PhD}^{1}$, Eid Rizk El Gammal, $\mathrm{PhD}^{2}$, Hadlaya Mosaad Eladl, $\mathrm{PhD}^{1}$ \\ ${ }^{1}$ Department of Physical Therapy for Surgery, Faculty of Physical Therapy, Cairo University, Giza; \\ ${ }^{2}$ Department of Oncosurgery, Faculty of Medicine, Al-Azhar University, Cairo, Egypt
}

\begin{abstract}
Objective To investigate the effect of aqua therapy resistance exercise on arm volume, pain, and shoulder range of movements in post-mastectomy lymphedema.

Methods This was a single-blind randomized controlled trial. Fifty eligible breast cancer survivors (median, 10 years after surgery) with lymphedema (median, $21 \%$ inter-limb difference) were assigned randomly to group A $(n=25)$ or control group $B(n=25)$. The study group underwent 60 minutes of aqua therapy exercise comprising of warm-up for 10 minutes, 40 minutes of strengthening exercises, and 10 minutes of cooling down, three times a week for 8 weeks. The control group underwent 60 minutes of land-based exercise three times a week for 8 weeks. Arm volume calculated by measuring the arm circumference, shoulder flexion, and abduction range of motion (ROM), and pain using a visual analog scale (VAS) were assessed at baseline and after 8 weeks of treatment.

Results There was a statistically significant difference in limb volume, shoulder flexion and abduction ROM, and VAS scores in favor of the study group $(\mathrm{p}<0.001)$ after 8 weeks of intervention. The mean \pm standard deviation for limb volume, shoulder flexion, abduction, and pain score were $2,108.71 \pm 200.97 \mathrm{~mL}, 169.68^{\circ} \pm 4.54^{\circ}, 150.44^{\circ} \pm 3.92^{\circ}$, and $3.16 \pm 1.1$ in the study group and $2,256.41 \pm 186.94 \mathrm{~mL}, 147.36^{\circ} \pm 5.32^{\circ}, 131.32^{\circ} \pm 4.38^{\circ}$, and $5.68 \pm 0.94$ in the control group, respectively.

Conclusion Adding aqua therapy resistance exercise to routine physical therapy might be more effective in decreasing the limb volume and pain intensity and improving ROM of the shoulder in postmastectomy lymphedema.
\end{abstract}

Keywords Aqua therapy, Resisted exercise, Postmastectomy lymphedema

Received July 6, 2020; Revised October 5, 2020; Accepted November 23, 2020; Published online April 14, 2021 Corresponding author: Hadaya Mosaad Eladl

Department of Physical Therapy for Surgery, Faculty of Physical Therapy, Cairo University, El-Tahrir St. Dokki, Giza 11432, Egypt. Tel: +20-1-0431-3596, Fax: +20-2-3761-7692, E-mail: hd mos@yahoo.com

ORCID: Khadra Mohamed Ali (https://orcid.org/0000-0002-8131-484X); Eid Rizk El Gammal (https://orcid.org/0000-0001-5990-7836); Hadaya Mosaad Eladl (https://orcid.org/0000-0002-4083-7070).

(c) This is an open-access article distributed under the terms of the Creative Commons Attribution Non-Commercial License (http://creativecommons.org/ licenses/by-nc/4.0) which permits unrestricted noncommercial use, distribution, and reproduction in any medium, provided the original work is properly cited. Copyright $\odot 2021$ by Korean Academy of Rehabilitation Medicine 


\section{INTRODUCTION}

Breast cancer-related lymphedema (BCRL) is a series of pathological conditions in which the protein-rich lymphatic fluid accumulates in the soft tissues due to lymphatic flow obstruction, leading to an increase in the arm circumference by $2 \mathrm{~cm}$ or more [1]. BCRL is more likely to develop 2 to 10 years after extensive surgery for breast cancer, involving dissection of the axillary lymph nodes, a large number of dissected lymph nodes, and mastectomy $[2,3]$. Typical symptoms of lymphedema include swelling, discomfort, pain, heaviness, tightness, stiffness of the shoulder, hypersensitivity, lack of sensation in the affected arm, and a decline in the overall daily functions [4].

Various therapeutic techniques can be used for treating BCRL, including complex decongestive physiotherapy (CDT), compression therapy, therapeutic exercises, and pharmacotherapy $[5,6]$. Physical therapy, along with various other interventions such as surgery, oral pharmaceuticals, low-level laser therapy, weight reduction, mesenchymal stem cell therapy, Kinesio taping, and acupuncture, might be effective in reducing lymphedema [7].

The National Lymphedema Network [8] noted that for patients with lymphedema, the three main forms of exercise should include aerobic, strength, and flexibility exercises, as exercise is part of a healthy lifestyle and is necessary for the successful management of lymphedema.

Stuiver et al. [9] stated that the risk of developing BCRL could be reduced by resistance training following breast cancer treatment. Few studies have investigated the role of prevention and efficacy of various exercises on the volume of the affected arm. Moseley et al. [10] reported that combined arm exercises and deep breathing have an immediate positive effect.

Aquatic therapy is used for the management of edema. It is based on the concept of the application of hydrostatic forces during immersion, along with exercises in thermo-neutral water, to activate the circulatory system [11]. There are different approaches to aquatic therapy, one of which is $\mathrm{Ai} \mathrm{Chi}$, an aquatic exercise sequence and slow-pace water-based activity with an objective of relaxation, balance, and pain management [12]. Tidhar et al. [13] described another slow aquatic therapy approach for BCRL. They reported that the therapy called aqua lymphatic therapy was effective in reducing the edema of the affected arm over a 14-month trial period.
There is minimal evidence regarding the beneficial effects of aquatic resistance exercise therapy on BCRL and shoulder range of motion (ROM). Therefore, this study aimed to examine the efficacy of aqua therapy resistance exercise in the management of BCRL in terms of improving shoulder joint pain and ROM. It provides a safe, effective, and non-invasive modality for the treatment of lymphedema and avoids invasive modalities and oral medications that cannot be tolerated over a long period due to their adverse systemic effects.

\section{MATERIALS AND METHODS}

\section{Study design}

This was a prospective, single-blinded, parallel-group randomized controlled trial conducted between July 2018 and November 2019.

\section{Participants}

Fifty female participants were selected from the National Cancer Institute in Cairo for treatment at the Eden Health Care, Cairo, for a period of 16 months. The Ethical Committee of the Department of Physical Therapy approved the study (No. P.T.REC/012/002615), and the trial was registered in a Clinical Trials.gov (ID: NCT04257643). The participants were examined for their eligibility to participate in the study; they signed the written consent form before enrolment.

The inclusion criteria were patients with a history of breast cancer for which they had undergone unilateral excision of the axillary lymph nodes and had mild to moderate degree or stage I-II lymphedema (a difference in circumference up to $2 \mathrm{~cm}$ compared to the other arm indicating mild lymphedema; a difference of $2-5 \mathrm{~cm}$ indicating moderate lymphedema) [14].

According to the International Society of Lymphology, early lymphedema is stage $\mathrm{I}$, which is an early onset of the swelling that is noticeable and subsides with elevation of the arm. Pitting might be present. Moderate lymphedema, which is stage II, is a consistent change in the volume of the arm with the presence of pitting. Elevation rarely reduces the swelling, and progressive tissue fibrosis occurs [15].

A detailed history of the patients was obtained, which included information about the affected side and type of surgery, the number of lymph nodes excised, the number 
of lymph nodes positive for cancer cells and the number of tumors, technique used for radiotherapy, systemic adjuvant treatment, duration of lymphedema, previous episodes of infection, and completion of adjuvant treatment except hormone therapy. Patients were excluded if they had any of the following: cancer recurrence, ongoing active oncological treatment, functional disorders impeding participation in the exercise programs, and open wounds in any part of the body.

\section{Randomization}

The participants were informed about the nature and advantages of the study, their right to withdraw or decline participation at any time, and the concealment of any data obtained. Coding of all data maintained anonymity. Patients with mild to moderate degrees of lymphedema were randomly assigned to two groups (A and B) using computer-generated randomization blocks. Randomization was generated by the first author who was not involved in data collection. After allocation, none of the patients dropped out of the study.

\section{Sample size calculation}

Sample size calculation was performed prior to the initiation of enrolment in the study using G*POWER statistical software (F tests, multivariate analysis of variance [MANOVA]: repeated measures within and between interaction measurements) according to the primary outcome, which was the limb volume measurement with an effect size of 0.41 and power of 0.8 , and a significance level of 0.05 . The calculation revealed that 50 would be an appropriate sample size for the study.

\section{Intervention}

Patients were allocated to the study group (groups A) and control group (group B). Each group had 25 female patients with BCRL, whose ages ranged from 37 to 67 years. The patients in the control group (group B) received a land-based exercise therapy program: 60 minutes per session, three sessions per week, spanning 8 consecutive weeks. The program was designed and modified from previous studies [16-19] to target all the upper limb muscles or movements that can be affected by BCRL. The first 10 minutes of the sessions comprised of warm-up exercises with a small soft-ball, fit-ball, mobility and stretching exercises (to target the upper arm muscles), horizontal extension, arm abduction to $135^{\circ}$, shoulder flexion involving elevation of the arm in the sagittal plane, and maintaining the stretching for 2 to 3 minutes [19], with the aim of improving the mobility and overall fitness. This was followed by $30-40$ minutes of exercise for muscle strength using dumbbells with free weights, which necessitates additional body control and increased joint motion, including all the upper limb muscles, dumbbells side rise for the abduction movement, and arm elevation. Biceps curls, elbow extension, external/internal rotations, protraction/retraction of the shoulder blades, wrist curls, ball pressing, and movement of the intrinsic muscles of the hand were also performed using dumbbells [20].

For the resistance exercise, the load was manipulated from $55 \%$ to $65 \%$ of $1 R M$ with a maximum of $15-20$ repetitions (1RM indicates the heaviest weight that the patient can lift with maximum effort in a single repetition) for 1 to 2 sets per exercise. To ensure the progressive nature of the training program, the resistance was increased in $5 \%-10 \%$ increments in the following week, if the participants were able to perform more repetitions than the RM specified during three consecutive sessions and reported no increase in arm symptoms [18].

The subjects were asked to perform diaphragmatic breathing exercises in combination with this exercise program; women were instructed to perform "belly" breathing before starting the exercise program. Tactile cueing is often necessary to facilitate diaphragmatic breathing. The patients were advised to take a deep breath through the nose, which inflated the abdomen like a balloon, and then exhale with a sigh from the mouth, repeating this three times, and then relax [21]. The last 10 minutes of the session were for cooling down following the stretching exercises for all the upper limb muscles [16].

The participants were informed about the advantages and instructed to use a compression garment during the exercise session, and they were asked to use the same type of garment to ensure similarity in the subject pressure $[22,23]$.

Patients in the intervention group (group A) underwent the same supervised land-based exercise program as the control group, but in water. The aqua therapy-resistance exercise program was conducted at a hydrotherapy pool at Eden Health Care, Cairo. The pool measured $8 \mathrm{~m} \times 15$ $\mathrm{m}$, with access via steps ranging from 1 to $1.5 \mathrm{~m}$ in depth. 
Interventions were primarily performed in the deeper section of the pool so that participants submerged their necks into the water. The thermoneutral temperature of the water was maintained at $30^{\circ} \mathrm{C}-32^{\circ} \mathrm{C}$. The subjects were instructed to adjust to the depth of the water in the standing position until the clavicles were completely submerged in water. The session involved warm-up for 10 minutes, workout for 40-45 minutes, and cooldown for 10 minutes. The patients were asked to stand in a swimming pool until the neck was submerged in water and were asked to perform arm exercises plus diaphragmatic breathing. Diaphragmatic breathing accelerates the clearance of lymphatic fluids during routine exercise. The duration of the exercise program was 60 minutes, three times a week, for 8 consecutive weeks [16]. Throughout the study period, none of the patients complained of infection or adverse effects of increased limb volume. A detailed assessment of the patients before each session was performed; if there was any area of wound or trauma, the sessions were postponed until complete healing. The subjects were instructed not to come to the aqua therapy sessions if they had an area of the skin with wound, infection, cuts, or bruises. In addition, pool cleanliness was ensured to avoid infection. All women who participated in the study were advised to take care of the skin of the affected limb according to the individual's clinical presentation and needs, keeping the limb elevated as much as possible; however, they were not advised any CDT or manual lymphatic drainage home program.

\section{Outcome measures}

The outcome measures included limb volume calculated by the circumference, ROM of the affected limb during shoulder flexion and abduction, and VAS for pain assessment. Assessment was conducted by a physiotherapist pre- and post-treatment.

\section{Limb volume measurement}

The primary outcome measure for evaluating the intervention was limb volume. This was performed by measuring the circumference of each segment of the limb between two consecutive circumferences as a truncated zone. The volume of the segment was calculated as $V=h\left(C 1^{2}+C 1 C 2+C 2^{2}\right) / 12 \pi$, where $V$ is the volume of the segment, $C 1$ and $C 2$ are the circumferences at the ends of the segment, and $h$ is the distance between them (seg- ment length) [24].

With the patient in a comfortable sitting position, a standard 1-cm retractable fiberglass tape was used for measuring the circumference. The limb was placed on a bedside table in the horizontal position and was fixed with an adhesive measurement strip from the axilla to the wrist to ensure consistency of the measurement at 10$\mathrm{cm}$ intervals. Measurements were initiated at the ulnar styloid process and subsequently at $10,20,30$, and $40 \mathrm{~cm}$ proximally [25].

\section{Shoulder joint ROM}

Active ROM for shoulder flexion and abduction in the standing position was evaluated using standard goniometry. Particular care was taken to prevent flexion of the trunk, which could potentially affect the shoulder ROM; the procedure was performed three times, and the mean reading was considered. Shoulder ROM was assessed before the treatment intervention and again at the end of 8 weeks [26].

\section{VAS for pain assessment}

Another outcome measure for treatment assessment was pain intensity. The visual analog scale (VAS) is a onedimensional measure used in adults, especially in those with chronic pain. The VAS is a constant scale consisting of a vertical (VVAS) or horizontal (HVAS) line, typically $10 \mathrm{~cm}$ long, anchored by two verbal descriptors, one for each intensity of the symptom. Instructions, time span for reporting, and verbal descriptor anchors were discussed in detail with the patients before measurement [27].

\section{Data analysis}

All statistical analyses were performed using IBM SPSS statistical package version 25 for Windows (IBM SPSS, Armonk, NY, USA). The mean, standard deviation, and frequencies were calculated for the descriptive statistics. Statistical significance was defined as $\mathrm{p}<0.05$. Characteristics of the subjects between the groups were compared using the t-test for continuous variables and the chi-square test for categorical variables. The Shapiro-Wilk test was used to check the normal distribution of data, and homogeneity between the groups was tested using Levene test for homogeneity of variances. Within- and between-group comparisons were performed using a mixed-design MANOVA. 
The partial squared eta was considered as the effect size. Post-hoc tests using Bonferroni correction were performed for subsequent multiple comparisons.

\section{RESULTS}

Sixty BCRL survivors were invited to join this study based on the eligibility criteria of BCRL following can- cer surgery. Ten of them did not complete the study and were excluded, as four of them did not meet the inclusion criteria and six declined to participate. Thus, the final number of participants was 50 , and they were allocated randomly to group A (study group) or group B (control group) (Fig. 1).

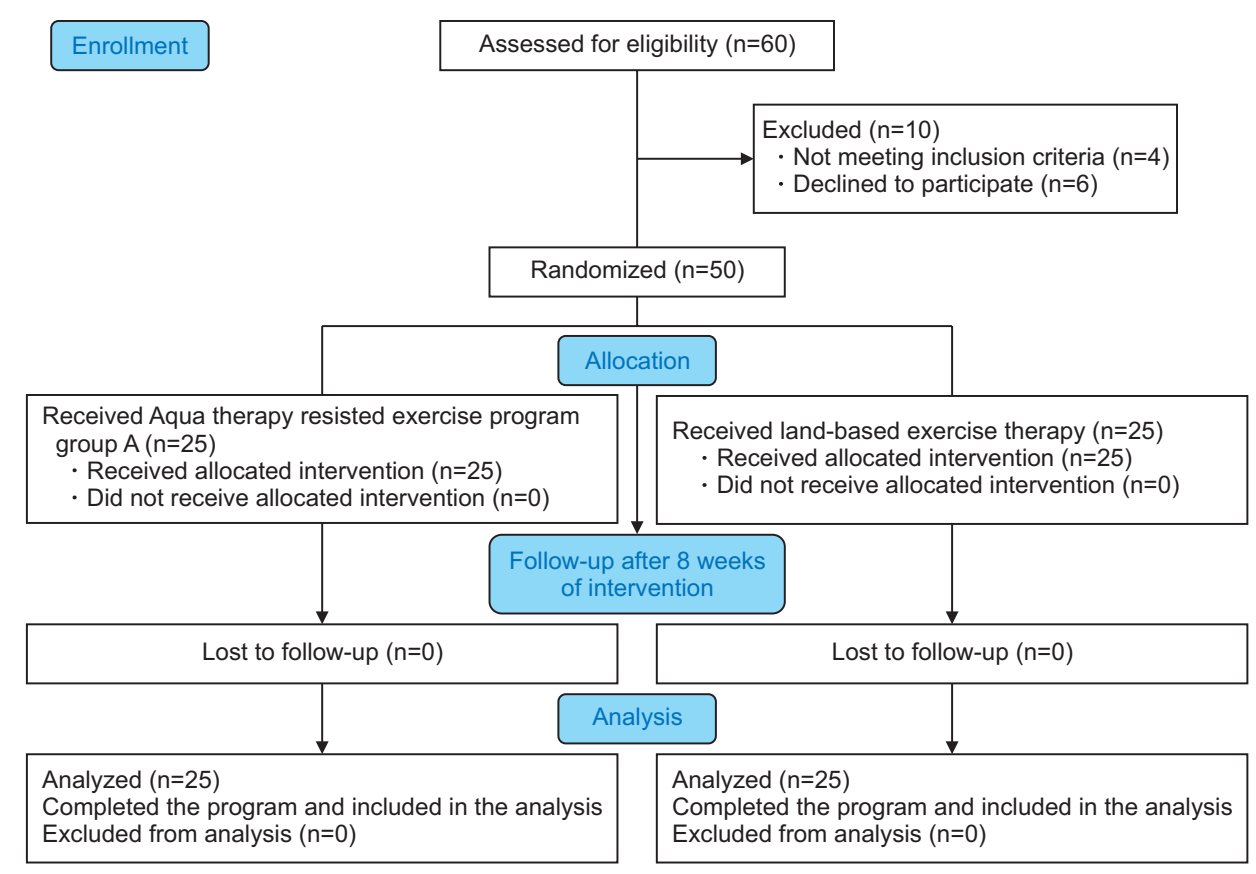

Fig. 1. Data analysis flow chart showing the progress of subjects at each stage of the clinical trial.

Table 1. Demographics of participants

\begin{tabular}{|lccc}
\hline & Group A & Group B & p-value \\
\hline Age $(\mathrm{yr})$ & $51.36 \pm 9.15$ & $49.84 \pm 8.57$ & 0.54 \\
\hline Weight $(\mathrm{kg})$ & $77.24 \pm 11.27$ & $77.88 \pm 12.65$ & 0.85 \\
\hline Height $(\mathrm{cm})$ & $160.2 \pm 6.15$ & $161.4 \pm 4.95$ & 0.45 \\
BMI $\left(\mathrm{kg} / \mathrm{m}^{2}\right)$ & $29.97 \pm 3.36$ & $29.78 \pm 4.00$ & 0.85 \\
\hline Number of lymph node resection & $11.88 \pm 3.98$ & $11.80 \pm 4.07$ & 0.94 \\
\hline Time since surgery (yr) & $2.64 \pm 1.37$ & $2.88 \pm 1.76$ & 0.59 \\
\hline Number of radiation therapy & $20.12 \pm 4.32$ & $21.92 \pm 4.42$ & 0.15 \\
\hline Number of chemotherapy & $6.76 \pm 2.43$ & $6.24 \pm 2.58$ & 0.46 \\
\hline Lymphedema stage & & & 0.77 \\
\hline Stage I & $11(44)$ & $10(40)$ & $15(60)$ \\
\hline Stage II & $14(56)$ & & 0.39 \\
\hline Type of surgery & & $9(36)$ & \\
$\quad$ Modified radical mastectomy & $12(48)$ & $16(64)$ & \\
\hline Partial mastectomy \& lymph node resection & $13(52)$ & & \\
\hline
\end{tabular}

Values are presented as mean \pm standard deviation or number (\%).

BMI, body mass index. 


\section{Characteristics of the subjects}

Subjects' characteristics of both groups are summarized in Table 1. There was no significant difference in the subjects' demographics and clinical data between the two groups $(\mathrm{p}<0.05)$.

Effect of treatment on the limb volume, shoulder ROM, and VAS

There was a significant interaction between the treatment and time (Wilks' $\lambda=0.01 ; \mathrm{F}_{(4,4)}=938.78, \mathrm{p}=0.001$, $\left.\eta^{2}=0.98\right)$. There was a significant main effect of time (Wilks' $\lambda=0.003 ; \mathrm{F}_{(4,4)}=3378.06, \mathrm{p}=0.001, \eta^{2}=0.99$ ) and treatment (Wilks' $\lambda=0.16 ; \mathrm{F}_{(4,4)}=59, \mathrm{p}=0.001, \eta^{2}=0.84$ ). Table 2 shows descriptive statistics of the limb volume, shoulder ROM, and VAS, as well as a significant level of comparison between the groups and a significant level of comparison between pre-and post-treatment in each group.

\section{Within-group comparison}

There was a significant decrease in the limb volume and VAS post-treatment compared to the pre-treatment measures in both the study and control groups ( $p>0.001)$. Furthermore, both groups showed a significant increase in the shoulder flexion and abduction ROM post-treatment compared to that before treatment $(\mathrm{p}>0.001)$ (Table 2).

\section{Between the groups comparison}

There was no significant difference in any of the parameters between the groups before treatment ( $p>0.05$ ). Comparison between the post-treatment parameters revealed a significant decrease in limb volume $(\mathrm{p}<0.01)$ and VAS $(\mathrm{p}<0.001)$ in group A compared to that in group $B(p<0.001)$ and a significant increase in the shoulder flexion and abduction ROM in group A compared to that in group $B(p<0.001)$ (Table 2).

\section{DISCUSSION}

The present study revealed a significant decrease in limb volume and VAS and increased ROM during shoulder flexion and abduction post-treatment compared with that at pre-treatment in both the study and control groups.

The results of the present study are in line with those of previous studies $[11,28-30]$. Previous systematic reviews

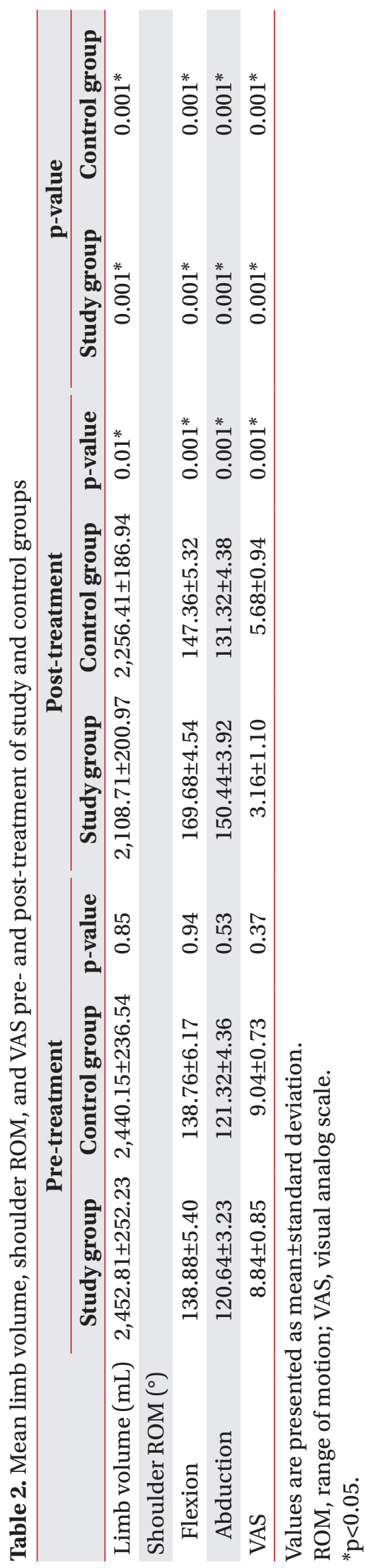


$[31,32]$ have shown that resistance exercise programs result in a reduction of the limb volume in patients with BCRL.

Aqua therapy programs vary widely in terms of the exercise mode, including resistance exercise, aerobic exercise, and flexibility exercise, in addition to variations in exercise intensity, frequency, and duration $[11,13,16]$. Furthermore, for the measurement of limb volume, many different tools of assessment are used to calculate the volume, using circumference measurement $[22,23]$ or water displacement [33], bioimpedance [22], or perometer [30].

In this study, we provided a modified, supervised aqua therapy-resistance exercise program to patients with BCRL, combined with diaphragmatic breathing exercises and the use of a pressure garment. We focused on resistance exercises for the upper arm muscles and increased the duration of resistance exercise to 30-40 minutes.

The increase in the ROM of shoulder flexion and abduction and decrease in the limb volume might be due to the hydrostatic pressure created by the aquatic exercise to reduce the residual volume of the arm through its squeezing action, thereby promoting central fluid flow. In addition, buoyancy and constant hydrostatic pressure allow the patients to move freely in water, providing additional muscle pumping to mobilize the lymphatic fluid [34]. Moreover, cancer survivors are often exhausted, and aquatic exercise is one way to minimize this exhaustion and decrease the pain caused by lymphedema [35].

A similar study conducted by Lindquist et al. [36] observed a decrease in lymphedema in the aqua therapy group without any change in the limb volume. This might be due to the short duration of strength exercise in their program; the duration of the session was only 10 minutes compared to 30-40 minutes in the present study. In contrast to the present study, the authors observed an improvement in the external rotation of the shoulder ROM in favor of the land-based exercise group.

In terms of limb volume reduction after aqua therapy, Tidhar and Katz-Leurer [11] conducted a study combining aqua lymphatic therapy in addition to self-massage and found an average immediate reduction of $92 \mathrm{~mL}$ in the limb volume as measured by the water displacement method. Moseley et al. [10] showed an immediate mean reduction of $52 \mathrm{~mL}$ after 10 minutes of arm exercise combined with breathing exercises. Johansson [37] demonstrated an immediate reduction of $32 \mathrm{~mL}$ after performing pool exercises at a water temperature of $28^{\circ} \mathrm{C}$ that included swimming, arm exercises with hand plates to increase water resistance, and jogging with arm movements.

In contrast to the findings of the present study, Fernandez-Lao et al. [16] showed an increase in limb volume in both water and land-based exercise-based programs compared to that in the control group. They explained that this was due to an increase in disuse and change in the limb using habits due to the fear of lymphedema.

According to Singh et al. [20], compression garments have been worn to mitigate the risk of short-term exacerbation. It is necessary to perform deep breathing when performing any lymphedema exercises. First, deep respiration is considered important to ensure adequate oxygen supply to the tissues when the body undergoes higher exertion than usual. Second, deep respiration changes the abdominal pressure that acts as a vacuum for the thoracic cavity, helping the lymphatic vessels to drain [38].

Diaphragmatic breathing during aquatic exercise has been shown to be effective in accelerating lymphatic drainage through the thoracic duct combined with slow water motion [39].

Pain reduction in the aqua therapy group might be attributed to the warmth and buoyancy of the water, which is believed to aid in the dissipation of allogenic chemicals and encourage relaxation of the muscles. Pain relief can also be due to a hydrostatic effect that increases peripheral edema and diminishes the sympathetic nervous system activity [40].

A significant observation in this study was the compliance of patients to the treatment program in both groups.

This study was limited by the following factors: the absence of long-term follow-up, so future studies are needed to assess the long-term follow-up for evaluation of the treatment modalities, also absence of evaluation of psychological status during the treatment period should be taken into consideration for future research, the final point is that different measurement tool might be necessary to achieve maximum reliability.

According to the present study, the aqua therapy resistance exercise program should be considered for the treatment of BCRL, as it is a safe, effective, and non-invasive method to decrease limb volume and consequently improve the ROM of the shoulder joint and decrease pain. 
However, further research is necessary to accurately design the optimum duration, intensity, and progression of the resistance exercise. In addition, different assessment tools are necessary to investigate the effect of aqua therapy exercise on the overall health of patients with BCRL.

In conclusion, based on the findings of this research, the aqua therapy resistance exercise demonstrated an effective role in decreasing limb volume and pain severity and increasing the ROM of the shoulder joint in patients with BCRL.

\section{CONFLICT OF INTEREST}

No potential conflict of interest relevant to this article was reported.

\section{ACKNOWLEDGMENTS}

We thank all our patients for their participation in the study.

\section{AUTHOR CONTRIBUTIONS}

Conceptualization: Ali KM, Eladl HM, El Gammal ER, Methodology: Ali KM, El Gammal ER, Formal analysis: Eladl HM, Ali KM. Project administration: AliKM, Eladl HM. Visualization: Ali KM, Eladl HM, El Gammal ER, Writing - original draft: Ali KM, Eladl HM, El Gammal ER. Writing - review and editing: Eladl HM, Ali KM, El Gammal ER. Approval of final manuscript: all authors.

\section{REFERENCES}

1. Armer JM, Stewart BR. Post-breast cancer lymphedema: incidence increases from 12 to 30 to 60 months. Lymphology 2010;43:118-27.

2. DiSipio T, Rye S, Newman B, Hayes S. Incidence of unilateral arm lymphoedema after breast cancer: a systematic review and meta-analysis. Lancet Oncol 2013;14:500-15.

3. Vieira RA, da Costa AM, de Souza JL, Coelho RR, de Oliveira CZ, Sarri AJ, et al. Risk factors for arm lymphedema in a cohort of breast cancer patients followed up for 10 years. Breast Care (Basel) 2016;11:45-50.

4. Lymphoedema Framework. Best practice for the management of lymphedema: international consensus.
London, UK: Medical Education Partnership (MEP) Ltd.; 2006.

5. Verbelen H, Gebruers N, Eeckhout FM, Verlinden K, Tjalma W. Shoulder and arm morbidity in sentinel node-negative breast cancer patients: a systematic review. Breast Cancer Res Treat 2014;144:21-31.

6. Shah C, Vicini FA. Breast cancer-related arm lymphedema: incidence rates, diagnostic techniques, optimal management and risk reduction strategies. Int J Radiat Oncol Biol Phys 2011;81:907-14.

7. Li L, Yuan L, Chen X, Wang Q, Tian J, Yang K, et al. Current treatments for breast cancer-related lymphoedema: a systematic review. Asian Pac J Cancer Prev 2016;17:4875-83.

8. National Lymphedema Network. Position statement of the National Lymphedema Network: exercise [Internet]. New York, NY: National Lymphedema Network; 2012 [cited 2021 Mar 1]. Available from: https:// klosetraining.com/wp-content/uploads/2015/05/ NLNpractices.pdf.

9. Stuiver MM, ten Tusscher MR, Agasi-Idenburg CS, Lucas C, Aaronson NK, Bossuyt PM. Conservative interventions for preventing clinically detectable upperlimb lymphoedema in patients who are at risk of developing lymphoedema after breast cancer therapy. Cochrane Database Syst Rev 2015;(2):CD009765.

10. Moseley AL, Piller NB, Carati CJ. The effect of gentle arm exercise and deep breathing on secondary arm lymphedema. Lymphology 2005;38:136-45.

11. Tidhar D, Katz-Leurer M. Aqua lymphatic therapy in women who suffer from breast cancer treatmentrelated lymphedema: a randomized controlled study. Support Care Cancer 2010;18:383-92.

12. Skinner EH, Dinh T, Hewitt M, Piper R, Thwaites C. An Ai Chi-based aquatic group improves balance and reduces falls in community-dwelling adults: a pilot observational cohort study. Physiother Theory Pract 2016;32:581-90.

13. Tidhar D, Shimony A, Drouin J. Aqua lymphatic therapy for postsurgical breast cancer lymphedema. Rehabil Oncol 2004;22:6-14.

14. Bar-Ad V, Cheville A, Solin LJ, Dutta P, Both S, Harris EE. Time course of mild arm lymphedema after breast conservation treatment for early-stage breast cancer. Int J Radiat Oncol Biol Phys 2010;76:85-90.

15. Executive Committee. The diagnosis and treatment of 
peripheral lymphedema: 2016 consensus document of the International Society of Lymphology. Lymphology 2016;49:170-84.

16. Fernandez-Lao C, Cantarero-Villanueva I, Ariza-Garcia A, Courtney C, Fernandez-de-las-Penas C, ArroyoMorales M. Water versus land-based multimodal exercise program effects on body composition in breast cancer survivors: a controlled clinical trial. Support Care Cancer 2013;21:521-30.

17. Johansson K, Hayes S, Speck RM, Schmitz KH. Waterbased exercise for patients with chronic arm lymphedema: a randomized controlled pilot trial. Am J Phys Med Rehabil 2013;92:312-9.

18. Garber CE, Blissmer B, Deschenes MR, Franklin BA, Lamonte MJ, Lee IM, et al. Quantity and quality of exercise for developing and maintaining cardiorespiratory, musculoskeletal, and neuromotor fitness in apparently healthy adults: guidance for prescribing exercise. Med Sci Sports Exerc 2011;43:1334-59.

19. Kilbreath SL, Refshauge KM, Beith JM, Ward LC, Lee M, Simpson JM, et al. Upper limb progressive resistance training and stretching exercises following surgery for early breast cancer: a randomized controlled trial. Breast Cancer Res Treat 2012;133:667-76.

20. Singh B, Newton RU, Cormie P, Galvao DA, Cornish $\mathrm{B}$, Reul-Hirche $\mathrm{H}$, et al. Effects of compression on lymphedema during resistance exercise in women with breast cancer-related lymphedema: a randomized, cross-over trial. Lymphology 2015;48:80-92.

21. Ambroza C, Geigle PR. Aquatic exercise as a management tool for breast cancer-related lymphedema. Top Geriatr Rehabil 2010;26:120-7.

22. Singh B, Buchan J, Box R, Janda M, Peake J, Purcell A, et al. Compression use during an exercise intervention and associated changes in breast cancer-related lymphedema. Asia Pac J Clin Oncol 2016;12:216-24.

23. Cormie P, Pumpa K, Galvao DA, Turner E, Spry N, Saunders C, et al. Is it safe and efficacious for women with lymphedema secondary to breast cancer to lift heavy weights during exercise: a randomised controlled trial. J Cancer Surviv 2013;7:413-24.

24. Casley-Smith JR, Casley-Smith JR. Modern treatment of lymphoedema. I. Complex physical therapy: the first 200 Australian limbs. Australas J Dermatol 1992;33:61-8.

25. Taylor R, Jayasinghe UW, Koelmeyer L, Ung O, Boy- ages J. Reliability and validity of arm volume measurements for assessment of lymphedema. Phys Ther 2006;86:205-14.

26. Norkin CC, White DJ. Measurement of joint motion: a guide to goniometry. 4th ed. Philadelphia, PA: F. A. Davis; 2009.

27. Hawker GA, Mian S, Kendzerska T, French M. Measures of adult pain: Visual Analog Scale for Pain (VAS Pain), Numeric Rating Scale for Pain (NRS Pain), McGill Pain Questionnaire (MPQ), Short-Form McGill Pain Questionnaire (SF-MPQ), Chronic Pain Grade Scale (CPGS), Short Form-36 Bodily Pain Scale (SF-36 BPS), and Measure of Intermittent and Constant Osteoarthritis Pain (ICOAP). Arthritis Care Res (Hoboken) 2011;63 Suppl 11:S240-52.

28. Jamison LJ. Aquatic therapy for the patient with lymphedema. J Aquat Phys Ther 2005;13:9-12.

29. Box R, Marnes T, Robertson V. Aquatic physiotherapy and breast cancer related lymphoedema. Proceedings of the 5th Australasian Lymphology Association Conference; 2004 March; Brisbane, Austrialia. p. 47-9.

30. Geigle PR, Kaufmann L. Lymphedema and aquatics with measurement by perometer (LAMP). Proceedings of the American Physical Therapy Association Combined Sections Meetings; 2006 Feb 2-5; San Diego, CA.

31. Nelson NL. Breast cancer-related lymphedema and resistance exercise: a systematic review. J Strength Cond Res 2016;30:2656-65.

32. Singh B, Disipio T, Peake J, Hayes SC. Systematic review and meta-analysis of the effects of exercise for those with cancer-related lymphedema. Arch Phys Med Rehabil 2016;97:302-15.

33. Schmitz KH, Ahmed RL, Troxel A, Cheville A, Smith $\mathrm{R}$, Lewis-Grant L, et al. Weight lifting in women with breast-cancer-related lymphedema. N Engl J Med 2009;361:664-73.

34. Wilcock IM, Cronin JB, Hing WA. Physiological response to water immersion: a method for sport recovery? Sports Med 2006;36:747-65.

35. Cantarero-Villanueva I, Fernandez-Lao C, Cuesta-Vargas AI, Del Moral-Avila R, Fernandez-de-Las-Penas C, Arroyo-Morales M. The effectiveness of a deep water aquatic exercise program in cancer-related fatigue in breast cancer survivors: a randomized controlled trial. Arch Phys Med Rehabil 2013;94:221-30. 
36. Lindquist H, Enblom A, Dunberger G, Nyberg T, Bergmark K. Water exercise compared to land exercise or standard care in female cancer survivors with secondary lymphedema. Lymphology 2015;48:64-79.

37. Johansson K. Controlled physical training for arm lymphedema patients. Lymphology 2004;37(Suppl):37-9.

38. Brennan MJ, Miller LT. Overview of treatment options and review of the current role and use of compression garments, intermittent pumps, and exercise in the management of lymphedema. Cancer 1998;83(12
Suppl American):2821-7.

39. Deacon R, de Noronha M, Shanley L, Young K. Does the speed of aquatic therapy exercise alter arm volume in women with breast cancer related lymphoedema? A cross-over randomized controlled trial. Braz J Phys Ther 2019;23:140-7.

40. Hall J, Swinkels A, Briddon J, McCabe CS. Does aquatic exercise relieve pain in adults with neurologic or musculoskeletal disease? A systematic review and meta-analysis of randomized controlled trials. Arch Phys Med Rehabil 2008;89:873-83. 